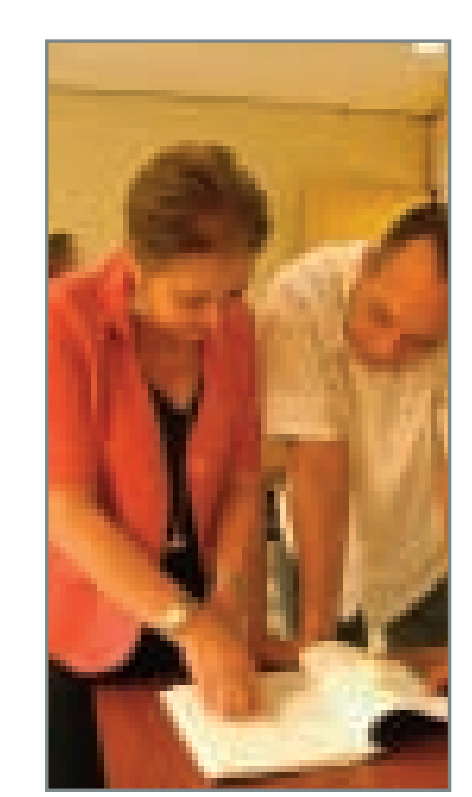

Los y las docentes han aprendido a mirarse a mismos, a interrogarse saber que todo esto potencia sus prácticas y puede iniciar diálogos interdisciplinarios. La enseñanza universitaria se ha cualificado y renovado a partir de estos procesos; posiblemente no carácter reproductivo, la docencia.

sos de investigación formativa, con el fin de fortalecer el pensamiento autónomo y reflexivo, en consonancia con planteamientos de la pedagogía crítica. Los y las docentes han aprendido a mirarse a sí mismos, a interrogarse y saber que todo esto potencia yira potencia sus prácticas y puede iniciar diálogos interdisciplinarios. La enseñanza universitaria se ha cualificado y renovado a partir de estos procesos; posiblemente no volverá a tener un carácter reproductivo, transmisivo, con énfasis en la docencia.

Esta construcción no se ha dado en un camino de rosas; las y los docentes participantes en calidad de estudiantes han experimentado diversas dificultades, que han sorteado y transformado en condiciones que analizan y mejoran desde su labor. Esta experiencia nos recuerda el concepto de resiliencia comado de la Fisica: Ios mejores metomado de la Fisica los mejores metales se funden a mayor calor y las mejores ostras, en la mayor presión. Los procesos sufridos y vividos en el proyecto por las y los docentes han buscado justo eso: pulir las más finas, hermosas y potentes aristas de la profesionalidad docente de quienes han cursado la Especialidad en Pedagogía Universitaria.

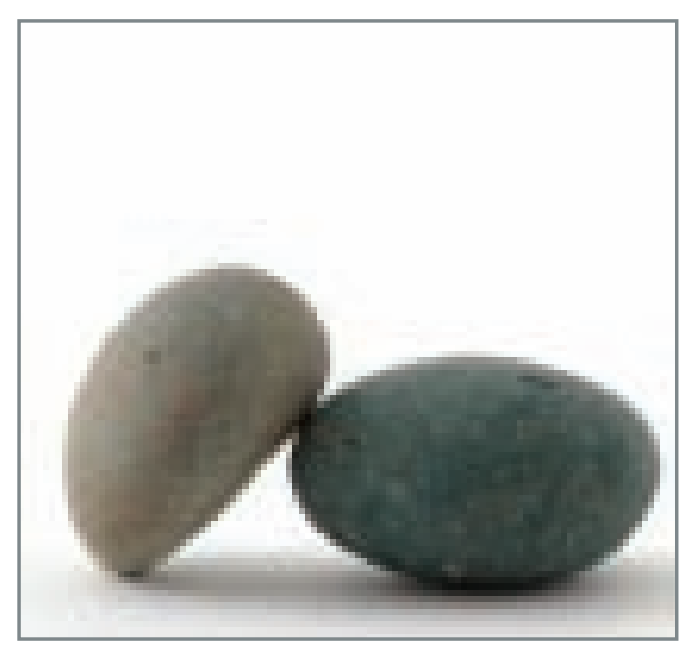

20 | Cuaderno de Pedagogía Universitaria
Referencias bibliográficas:

Cassany, D. (1993). Reparar la escritura. Didáctica de la corrección de lo escrito. Barcelona: Graó.

Elliot, J. (1991). El cambio educativo desde la investigación - acción. Morata: Madrid

Freire, P. (1970). Pedagogía de oprimido. Bogotá: Siglo XXI

Freire, P. (1971). Educación como práctica de la Libertad. Bogotá: Siglo XXI

Freire, P. (1997). Pedagogía de la Esperanza. Bogotá: Siglo XXI

Gimeno, J. (1988). El currículo: una reflexión sobre la práctica. Morata: Madrid

Morin, E. (1999). La cabeza bien puesta. Repensar la reforma, reformar el pensamiento. Bases para una reforma educativa. Buenos Aires: Nueva Visión, SAIC

PUCMM, Centro de Desarrollo Profesoral. (2007). Propuesta curricular de la Especialidad en Pedagogía Universitaria. Santiago: PUCMM

Zabalza, M. (2002). La enseñanza universitaria: el escenario y sus protagonistas. Madrid: Narcea

\section{Proceso de normalización de una prueba psicométrica en el Departa- mento de Orientación de la PUCMM}

por Berta Tavares Hernández*

Ciertamente, el asesoramiento vocacional desde la Escuela tiene una amplia tradición en el nivel de enseñanza secundaria y en los primeros años de la enseñanza superior, sobre todo en el mundo anglosajón, en el que los consejeros han ido actuando no sólo como psicólogos de despacho dedicados a la consulta individual, sino interviniendo activamente en la programación de actividades vocacionales complementarias al currículum escolar y en la movilización de los recursos de la comunidad. A la postre, se entiende el hecho vocacional como una dimensión educativa de primer orden en el desarrollo individual.

Rivas, Psicología Vocacional: Enfoques del Asesoramiento.

(1993, p. 33).

El Departamento de Orientación de la PUMM en el campus de Santiago es una dependencia del Decanato de Estudiantes. Se ofrecen los servicios de consejería/asesoría, información, ubicación, evaluación y seguimiento a los

estudiantes y se investiga el impacto que tienen estos servicios para poder mejorarlos. Para los fines de este artículo nos enfocaremos en la evaluación, que consiste en la aplicación de pruebas psicométricas que se toman a voluntad del alumno o por requerimiento de otras unidades de la Universidad.

Los nombres y áreas de evaluación de estas pruebas son: "D-48", valora la Inteligencia General; "PMA" evalúa las Habilidades Generales; "16FP", valora los dieciséis Factores de la Personalidad; "EMT", evalúa el método de trabajo y técnicas del estudiante: "MMPl", Inventario Multifásico de Personali. o de MAP", o Cuestionario de Adaptación para Adultos y "KUDER", que mide los intereses vocacionales. Es esta última prueba la que se está sustituyendo.

Uno de los objetivos generales, trazados en el documento del Plan de Desarrollo Estratégico 2006-2009 de Departamento de Orientación, tiene que ver con fortalecer la calidad del proceso de evaluación del estudiante.

Para lograr este objetivo, los miembros

Egresada de la carrera de Educación con concentración en Orientación Escolar. Postgrado en Geren-

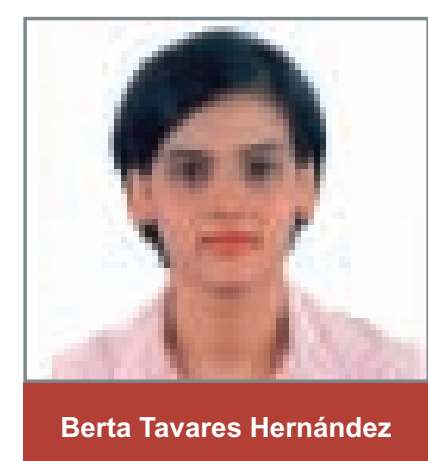

En este artículo se hace un recuento del proceso de normalización de la prueba psicométrica "Inventario de Intereses y Preferencias Profesionales, versión Revisada (IPP- R)" que se visada (IPP R) que se ha partamento de Orientación de la PUCMM, en el campus de Santiago. El uso de esta prueba mejorará los servicios del Departamento en el área de evaluación vocacional, debido a la actualización de las carreras sugeridas, la disminución del tiempo de aplicación y la simplificación de su corrección. 


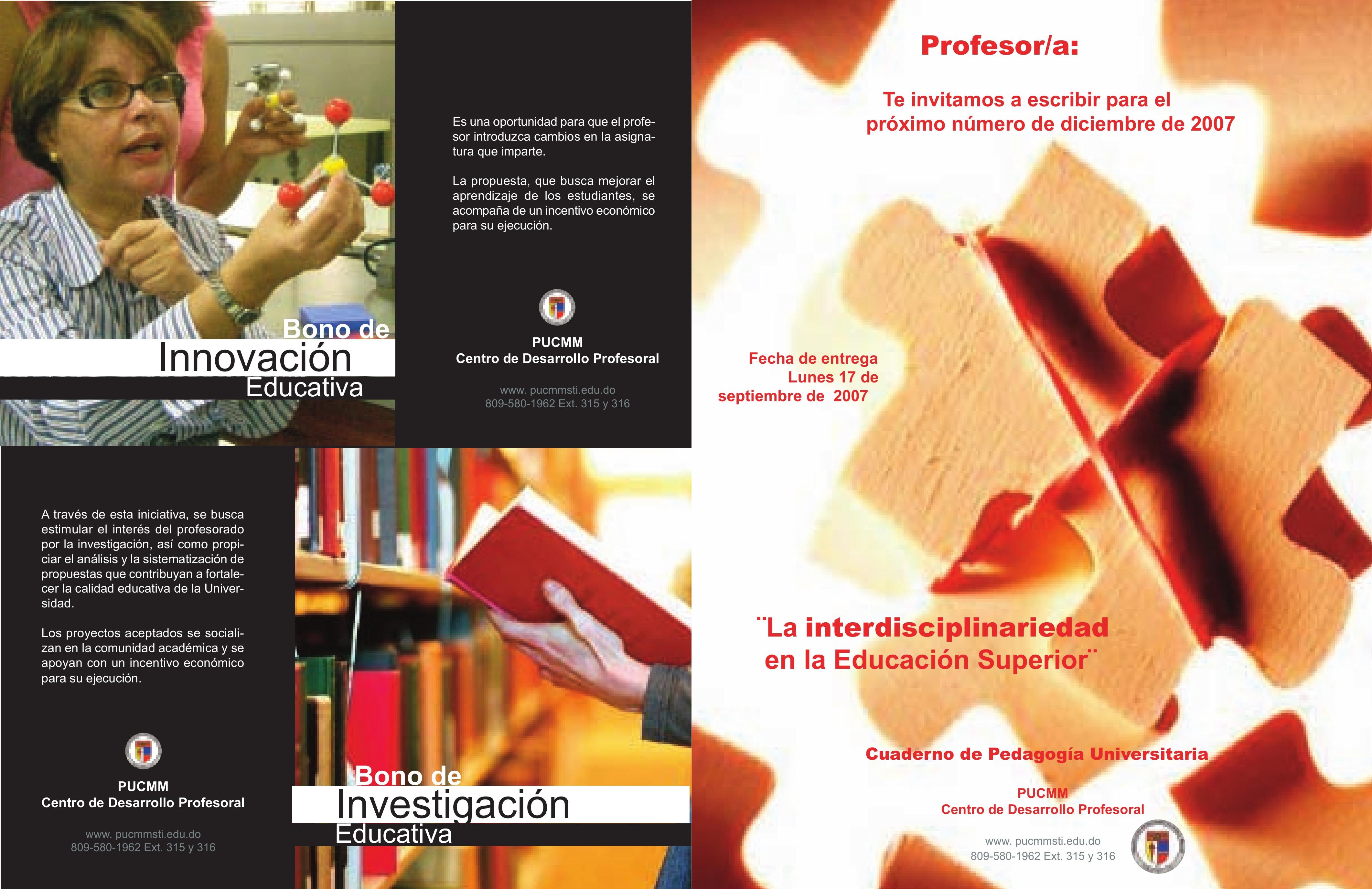


abe mencionar la imporancia de utilizar las norm que se adapten a nuestra cultura pues, de lo contrario, los resultados de prueba no serían adecuados para el estudiante. Además, se debe tomar en cuenta que en la adaptación hay que mantener la constante renovación, dados los cambios en el ámbito de intereses vocacionales orientados hacia el desempeño laboral del mercado

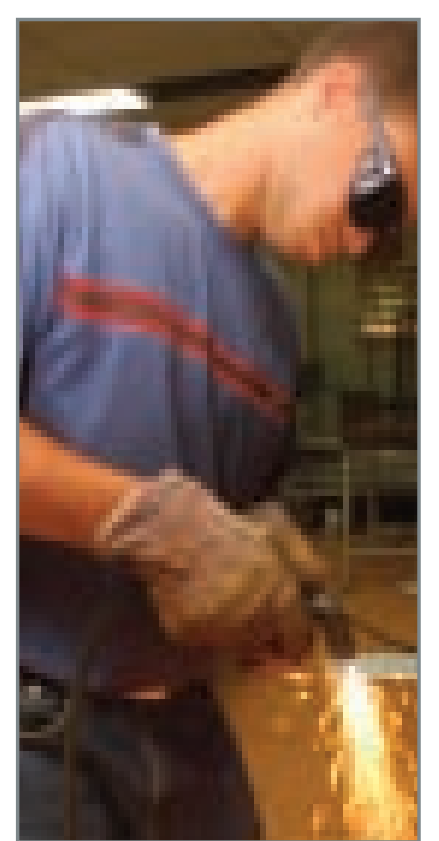

del Departamento ${ }^{1}$ optamos por reflexionar sobre las necesidades y priordades del proceso de aplicación de pruebas psicológicas en el contexto de as exigencias de la actualidad. En este sentido, se llevaron a cabo Ia guientes acciones:

- Observamos la necesidad de actualizar las pruebas psicométricas, priorizando el área de los intereses vocacionales, cionales, a sea, la sustitución de

Hicimos contacto con instituciones de distribución y venta de pruebas psicológicas en distintos países.

- Analizamos los catálogos de pruebas y las comparamos con las que hay en existencia en el Departamento de Orientación.

- Llegamos a un acuerdo para la adquisición de una prueba actualizada que respondiera a las necesidades de la población estudiantil de esta Institución.

Seleccionamos la prueba "Inventario de Intereses y Preferencias Profesionales, Revisado (IPP-R)" para ser adaptada a población estudiantil que se atiende.

Adquirimos la prueba a la compañía Desarrollo S.A., asociada con TEA Ediciones S.A., casa matriz y distribuidora de la prueba IPP-R, ubicada en Madrid España. (http://www.teaediciones.com)

Una vez elegida la prueba, se tomó en cuenta que la misma se ajustara a las características de nuestra población. Para esto se debe pasar por un proceso riguroso que nos deje medir con precisión y validez la conducta del estudiante en un momento dado sobre e

1 Los miembros del Departamento de Orientación en el campus de Santiago son: Rosa Liriano de 1 Los miembros del Departamento de Orientación en el campus de Santiago son: Rosa Liriano de
Batista, Idelise Sagredo, Orquidea Grullón, Maria Elizabeth Frómeta, Mercedes Carmen Estévez, Gio-
vanny Heredia y Berta Tavares.

área seleccionada, y que permita recopilar, analizar e interpretar los dato que se estudian.

Es aquí cuando entra en escena la necesidad de normalizar, validar y determinar la confiabilidad del IPP-R. Ya que esto conlleva tiempo y dedicación, decidimos iniciar el proceso de normalización de la prueba.

Ahora bien, ¿qué es la normalización de una prueba psicométrica? Herran 2000) lo explica muy bien a continuación:

Las normas de un examen psicológico se refieren a la ejecución típica realizada en ese examen por la muestra de estandarización. Específicamente, la normalizació consiste en el promedio aritmético la media y la desviación estándar derivados de la muestra de estandarización que permiten efectuar la conversión de las puntuaciones brutas o directas de la prueba a una escala de puntuaciones derivadas ( $Z$, Percentiles, $T$, etc.) (p. 27)

Cabe mencionar la importancia de utilizar las normas que se adapten nuestra cultura pues, de lo contrario, os resultados de la prueba no sería adecuados para el estudian se debe tomar en cuenta que en la adaptación hay que mantener la renovación, dados los cambios en el ámbito de intereses vocacionales orientados hacia el desempeño laboral del mercado Como dice Herrans (2000), "La 22 | Cuaderno de Pedagogía Universitaria población a otra población distinta es frecuente en países latinoamericanos y del Caribe" (p. 27)

La normalización de cualquier prueba psicométrica debe seguir un proceso que incluye varias fases. A continuación Herrans (2000) las distribuye de esta manera:

- La estandarización del examen consiste en la construcción de la forma final del mismo examen y el desarrollo de un manual de procedimientos uniforme para administrar y valorar los resultados de ese examen.

La selección de una prueba representativa de la población para la cua se construye el examen.

- La administración del examen a la - La administración del examen a la muestra de estandarización (la muestra representativa) siguiendo los procedimientos uniformes estipulados en el manual de instrucción del examen.

- El establecimiento de las normas: La desviación estándar de los puntajes obtenidos por la muestra de estandarización, que permita luego la conversión de los puntajes brutos a puntajes estándar.

- El proceso de conversión de los puntajes brutos (total de las respuestas correctas obtenidas por cada sujeto en ( Un puntaje bruto en un examen no tiene significado a menos que se le compare con alguna vara o criterio específico. (pp. 25-26)

Este proceso fue iniciado formalmente con el IPP-R en el periodo 2-20052006, para ref dedes y pionidades del Departamento. Se pretende finalizar la normalización en el transcurso del período académico enero-mayo 2007
Para comprender las características que favorecieron la selección, debemos hacer una comparación entre ambas pruebas psicométricas, KUDER e IPPR. La pruba KUDER Ku KR IPP Frederic Kuder primer récord de preferencias en el ño 1939, implementándose en el país 1970. Esta prueba se aplica de manera individual o colectiva adolescentes y adultos, la cual tiene una duración aproximada de 30 a 60 minutos. co corrección de la prueba sú computarizada, pero su software con la mayoría de las plataformas tecnológicas actuales. Además, es algo tedioso para quien lo realiza. Ella evalúa 10 áreas, las cuales son: mecánica científica, persuasiva, artística, literaria, musical, administrativa, servicio social aire libre y cálculo.

En el año 1984, Dioda Reyes de Olivo realizó en esta Universidad la investigación "Normalización del Test de Intereses Vocacionales KUDER", la cua se utiliza hoy en día en muchas instituciones educativas de nuestro país. Las matrículas se usaron a partir de lo alumnos del año 1977 hasta el 1983 inclusive. El estudio se encuentra en os archivos del Departamento de Orientación de la PUCMM, donde la muestra seleccionada representa todas las carreras de la Institución en ese entonces. El manual pertenece a la compañía de Evaluaciones Psicológicas Sistémicas (EPSI).

La prueba psicométrica seleccionada PP-R, cuyas siglas significan Invenario de In sio-nales, es la Versión Revisada en e año 2004 por la misma autora de la prueba, María De la Cruz López, en Es-

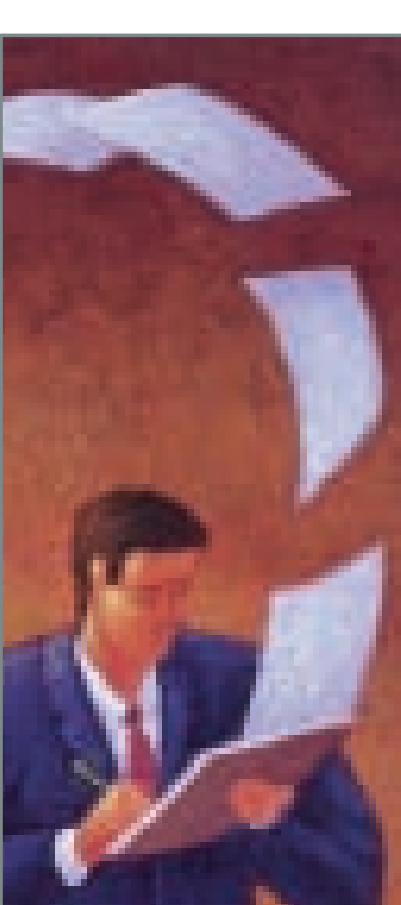

El concepto de estandarización es definido por Anastasi \& Urbina (1998) como

la igualdad de los procedimientos en la aplicación y calificación de la prueba para asegurar la uniformidad de las co dad de las condiciones de la misma. Además, ella se extiende a los materiales exactos que deben emplearse, los límites de tiempo, las instrucciones orales, las demostraciones previas, las formas de manejar las dudas de los examinados y cualquie otro detalle de la situación valuativa (p. 6).

Cuaderno de Pedagogía Universitaria $\quad$ | 23 


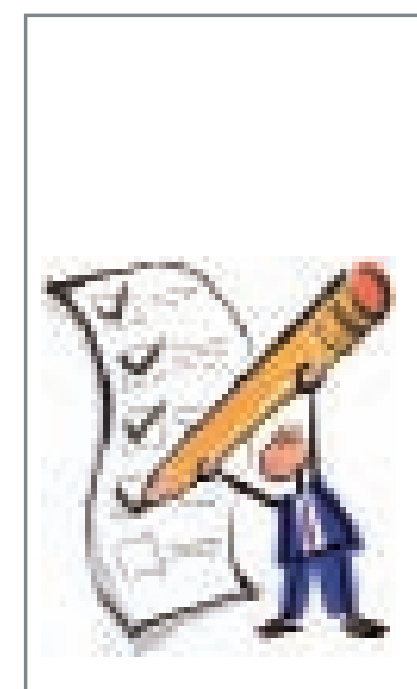

Si se volviera a normalizar la prueba KUDER, ésta podría seguir siendo utilizada, dado que es una de las mejores herramientas par mediciones vocacionales. Se decidió, la prueba IPP-R por el desfase de las carreras sugeridas, comparadas con las existentes en el mercado actual, así como para acortar el tiempo de aplicació y simplificar la corrección de la misma.

paña. Tiene un tiempo de aplicación de 20 a 25 minutos. Ella cuenta con 15 campos profesionales: científico, técnico, sanitario, humanidades, jurídicosocial,comunicación-información psicopedagógico, empresarial-administrativo-comercial, in trativo-comercia, infomatica, agrarioa gropecuario-ambiental artístico-plástico-artesanía, artísticomusical-espectáculos, fuerzas armadas-seguridad-protección, deportivo, turismo y hotelería. Su corrección se realiza utilizando un pin en www.e-perfil.com, en donde consume un uso por sujeto evaluado.

Si se volviera a normalizar la prueb KUDER ésta podría seguir siendo utilizada, dado que es una de las mejores herramientas para mediciones vocacionales. Se decidió, en cambio, usar la prueba IPP-R por el desfase de las careras sugeridas, comparadas con las existentes en el mercado actual as como para acortar el tiempo de aplicación y simpificar la correccion aplcación y simplificar la corrección de la misma.

Para normalizar el IPP-R, se contó con la asistencia de las estudiantes de térPsicología Organizacional, Miguelina Cruz y Pamela Lomba, las cuales tomaron, a su vez, este proyecto para su tesis de licenciatura. Ellas han sido asesoradas di-

Referencias bibliográficas:

Anastasi, A. \& Urbina, S. (1998). Tests Psicológicos. (7ª ed.). México: Prentice Hall.

Herrans, L. (2000). Psicología y Medición. (2ª ed.). México: McGraw-Hill.

Rivas, F. (1988). Psicología Vocacional: Enfoques del Asesoramiento. ( $2^{\mathrm{a}} \mathrm{ed}$.) Madrid: Morata.

24 I Cuaderno de Pedagogia Universitria rectamente por Giovanny Heredia psicólogo del Departamento de Orientación y, en diferentes momentos del proceso, por los los colegas Ysidro Lopez y Kiero Guerra. Quien escribe este artículo está a cargo de la gestión conexión con Desarrollo S. A., la casa distribuidora de la prueba.

De nada nos serviría que dejáramos por concluido este proyecto con la normalización de la prueba IPP- $R$, ya que aún nos falta determinar su validez y confiabilidad. Tenemos un reto por delante para poder cumplir con el objetivo trazado.

Normalizar la prueba IPP-R traslada e area de la medición y la evaluación psicológica de nuestro país al nuevo siglo. Además, ésta deja entrever la ardua labor que se tiene por delante y las expectativas de que esta iniciativa motive a otros a comprender la importancia de tomar en cuenta estos procesos realizarlos en sus ámbitos laborales.

el Departamento de Orientación se aspira a continuar con las demás pruebas para ofrecer un mejor servicio a los estudiantes de la PUCMM.

\section{Derecho de las Tecnologías de la In- formación y la Comunicación en la Universidad: ¡Tiempo de investigar! por Dariel Suárez*}

A inicios del Siglo XXI, el desarrollo de las nuevas tecnologías de la información vinculadas a la revolución de las telecomunicaciones ha planteado nuevos desafíos para el derecho. E advenimiento del mundo digita provoca la aparición de circunstancias totalmente nuevas que impiden en ocasiones tanto la aplicación de instrumentos jurídicos tradicionales como su adaptación al nuevo medio; exigiendo, en consecuencia, nuevas formulaciones específicas por parte del orden jurídico.

Carbonell, Sociedad de la Información y derechos humanos de la cuarta generación, 2004. constantes y trascendentales cambios, no puede concebirse la enseñanza de Derecho sin investigación. Fruto del desarrollo de las TIC ${ }^{1}$ y su implementación en todos los ámbitos de nuestra sociedad, podemos afirmar que el Dere-
Hoy en día, en una sociedad de cho se enfrenta a grandes desafíos y ha de ser objeto de profundos cambios, sobre todo en la manera de entenderse y enseñarse.

Es ya normal, en nuestro entorno universitario, escuchar hablar sobre necesidad de incorporar las TIC a la formación docente y de enriquecer con ellas los procesos de aprendizaje, pero no debemos dejar a un lado la reflexión sobre las implicaciones jurídicas que tiene su uso, sobre todo en este mismo entorno, y la necesidad de investigar constantemente para poder dar respuesta a los principales problemas que pueden surgir.

Se trata de la urgente necesidad de que, en la Pontificia Universidad Católica Madre y Maestra (PUCMM) convivan armoniosamente las TIC y el Derecho. Este es un tema que desde hace muchos años viene discutiéndose en los países desarrollados y que hoy es tomado muy en serio en la mayoría de sus universidades. ${ }^{2}$ Prueba de ello

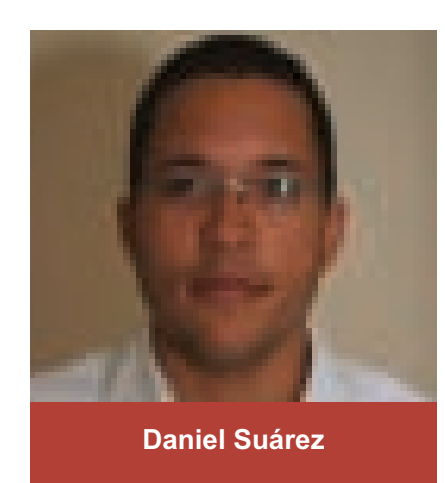

Resumen:

Eresente artículo busca oncienciar sobre la urgente necesidad de promover la investigación en e ambito del Derecho de las Tecnologías de la Información y la Comunicación en la Universidad. Esto así, dadas las implicaciones juídicas del uso de las Tecnologías de la Información y la Comunicación (TIC) en nuestra sociedad moderna y la necesidad, para el Derecho, de adoptar nuevas estrategias $y$ perspectivas para hacer frente a los retos a los que se enfrenta a consecuencia de ello. * Licenciado en Derecho, Mastría en Derecho de las Telecomunicaciones y las Tecnologias de la Infor-
mación en la Universidad Carlos III, en Madrid. Profesor del Departamento de Ciencias Juridicas de la

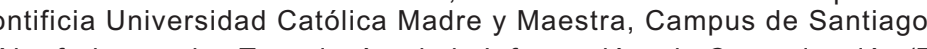

Al referirnos a las Tecnologías de la Información y la Comunicación (TIC) las queremos precisar como

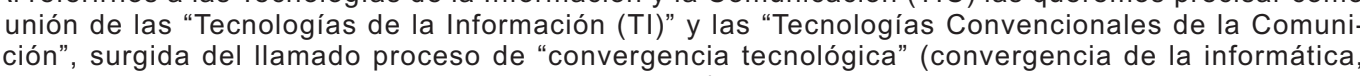
as telecomunicaciones, la electronica y los contenidos).

2 Son muchas las universidades que cuentan con centros especializados en la investigación del Dere-
cho de las TIC. Entre estos, cabe citar: en Europa: el Instituto de Informática Jurídica de la Universidad Pontificia Comillas y el Équipe de Recherche Informatique \& Droit de la Universidad Montpellier I; en los Estados Unidos, el Berkeley Center for Law \& Technology y, en
Derecho Informático de la Universidad de Chile, entre otros. 